obese patients, even in the high-risk older population. Quality of life might also improve considerably through amelioration of comorbid conditions.

Original article Perry CD et al. (2008) Survival and changes in comorbidities after bariatric surgery. Ann Surg 247: 21-27

\section{Plasma albumin mRNA levels might predict post-transplant recurrence of HCC}

Post-transplant recurrence of hepatocellular carcinoma (HCC) is common, despite routine use of patient-selection criteria based on pretransplantation radiological findings. HCC recurrence in the liver graft is probably caused by cancer cells circulating in the blood, and levels of cancer-derived nucleic acids in plasma are thought to reflect this circulating tumor-cell burden.

Cheung and colleagues analyzed prospectively collected data from 72 patients who underwent liver transplantation for HCC at Queen Mary Hospital, Hong Kong, between 1997 and 2005. They extracted RNA from plasma samples obtained from each patient immediately before surgery, as well as from 10 healthy control individuals and 10 non-HCC liver-transplant recipients. Perhaps surprisingly, albumin messenger RNA (mRNA) levels (but not alfafetoprotein mRNA levels) were significantly associated with post-transplant HCC recurrence $(P=0.029)$. The researchers calculated that the ability of preoperative plasma albumin mRNA levels to predict posttransplant HCC recurrence was superior to that of preoperative radiological assessments of tumor size and number, and comparable to that of pathological findings of vascular invasion (overall accuracy $71 \%$ for plasma albumin mRNA, compared with $74 \%$ for vascular invasion).

The authors note that preoperative assessments of vascular invasion require tumor biopsy, which is a risky procedure. By contrast, plasma albumin mRNA levels are simple and easy to measure. The authors have initiated a multicenter, prospective study in a large group of patients to evaluate their findings further.

Original article Cheung ST et al. (2008) Albumin mRNA in plasma predicts post-transplant recurrence of patients with hepatocellular carcinoma. Transplantation 85: 81-87

\section{Patients misperceive the risks and benefits of infliximab therapy for IBD}

Infliximab is a chimeric, monoclonal antibody to tumor necrosis factor; this therapy induces or maintains remission of Crohn's disease and ulcerative colitis in $\sim 60 \%$ of cases, even if other treatments have failed. Serious adverse effects of infliximab therapy are rare, but include lymphoma and life-threatening infections.

Siegel and colleagues analyzed $165 \mathrm{com}-$ pleted questionnaires that evaluated perceived risks and benefits of infliximab therapy. Respondents were patients, or parents of patients, with IBD who attended patient-education symposia in Boston, MA and Chicago, IL. Respondents overestimated the benefits of infliximab $(59 \%$ expected a remission rate $>50 \%$, and $18 \%$ expected a remission rate $>70 \%$, at 1 year). They also underestimated its risks: $37 \%$ denied a link between infliximab and lymphoma, and $67 \%$ thought that infliximab conferred a lymphoma risk less than double that of the general population (the observed risk is 20 -fold that of the general population). When presented with a hypothetical new therapy for IBD that had a risk profile corresponding to that of infliximab, $64 \%$ of respondents said they would refuse such treatment, even though one-third of this group were currently taking or had taken infliximab. Parents of patients were less optimistic than patients with regard to both risks and benefits of infliximab therapy.

Many patients with IBD could be taking medications that do not align with their risk-benefit preferences, say Siegel and colleagues. They urge physicians to communicate more effectively with their patients.

Original article Siegel CA et al. (2008) Patient perceptions of the risks and benefits of infliximab for the treatment of inflammatory bowel disease. Inflamm Bowel Dis 14: 1-6

\section{Cannabis use is a risk factor for fibrosis in patients with chronic HCV infection}

HCV-related cirrhosis and liver cancer rates are predicted to increase in the US in the next 10 years. Several risk factors - notably male sex, increased duration of HCV infection, older age at initial infection, heavy alcohol intake and 